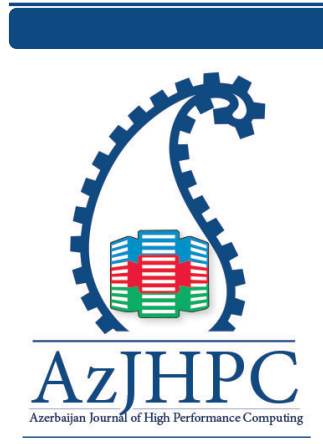

${ }^{*}$ Correspondence: Nigar Ismayilova, High

Performance Computing

Research Advance

Center, Department of

General and Applied

Mathematics, Azerbaijan

State Oil and Industry

University, Baku,

Azerbaijan,

nigar.ismailova@asoiu. edu.az

\title{
Parallel numerical method of an inverse problem of double-phased filtration
}

\author{
Araz Aliev, Khanlar Hamzaev, Nigar Ismayilova, Eldar Jahangirbayov, \\ Farid Jafarov, Rahman Mammadov \\ High Performance Computing Research Advance Center, Department of General and \\ Applied Mathematics, Azerbaijan State Oil and Industry University, Baku, Azerbaijan \\ alievaraz@azjhpc.org,xan.h@rambler.ru,nigar.ismailova@asoiu.edu.az,craid652@gmail. \\ com,feridcefer@hotmail.com,mammadli.kenta@gmail.com
}

\section{Abstract}

Was investigated the process of the displacement of oil with water in the horizontally located two-dimensional layer, which is described with the double-phased filtration model of incompressible liquid in non-deformable porous media. Within this model was set inverse problem for the definition flow rate of exploitative wells. Meanwhile, as additional conditions are set downhole pressure in injection wells. For the numerical solution of the problem, firstly was conducted discretization by time. As a result original problem comes down to two independent problems which are solved sequentially in each layer of time: the inverse problem for the definition of pressure distribution and flow rate of exploitative wells and forward problem for the definition of saturation of displacing phase. For the solution of the forward problem was offered particular fission, which gives an opportunity parallelization of received differential problems. In the base of the offered numerical method were carried out experimental results for model tasks. For parallelization of computing, processes was applied Open MP technology.

\section{Introduction}

Water flooding - injection of the water to the media by individual ascension wells is one of the leading technologies for the exploitation of oil reserves. By this method, it is possible to solve two interrelated problems: sustaining the pressure of the media, which helps to not to fall the debits of extractive wells, at the same time provides the displacing of the oil from the media to extractive wells. It should be mentioned that the role of the water in the process of displacement is challenging. In the one hand as displacing agent, water lets to increase the coefficient of oil production, in the other hand by rushing to wells and watering them, decreases the effectivity of exploitation of the oil field. By this reason for control of the processes of oil displacement from the media by the water, for projecting of development systems and during exploitation of oil fields was widely applied mathematical modeling methods. By application of modeling methods, it is possible to explore different development methods, to evaluate the effectivity the various methods to influence media, to predict the behavior of the oil field. 
For modeling of the oil displacement process from the media by water is used following equations system, which includes continuity equations of filtration of oil and water flow:

$$
\begin{aligned}
& \frac{\partial \rho_{w} S_{w} \phi}{\partial t}+\operatorname{div} \rho_{w} \vec{u}_{w}=0, \\
& \frac{\partial \rho_{0} S_{0} \phi}{\partial t}+\operatorname{div} \rho_{0} \vec{u}_{0}=0,
\end{aligned}
$$

equation of the liquid flow (generalized rule of Darcy)

$$
\begin{gathered}
\vec{u}_{w}=-\frac{k k_{w}\left(S_{w}\right)}{\mu_{w}}\left(\operatorname{grad} P_{w}-\rho_{w} \vec{g}\right), \\
\vec{u}_{0}=-\frac{k k_{0}\left(S_{w}\right)}{\mu_{0}}\left(\operatorname{grad} P_{0}-\rho_{0} \vec{g}\right),
\end{gathered}
$$

law of performance of the porous media and liquids

$$
\phi=\phi\left(P_{w}, P_{0}\right), \rho_{w}=\rho_{w}\left(P_{w}\right), \rho_{0}=\rho_{0}\left(P_{0}\right),
$$

where $S_{w}$ - is the saturation of displacing phase (water), $S_{o}-$ is the saturation of displaced phase (oil), $\vec{u}_{w}, \vec{u}_{0}-$ are speeds of filtration of displaced and displacing phases, $P_{w}, P_{0}$-pressures in water and oil phases, $k_{w}\left(S_{\mathrm{w}}\right), k_{o}\left(S_{w}\right)$ - relative phase porosities of the water and oil, $\mu_{w}, \mu_{o}$-viscosity of the water and oil, $k$ - the coefficient of absolute porosity, $\phi$ - coefficient of porosity, $\rho_{w}, \rho_{0}$-densities of the water and oil, $\vec{g}$ - acceleration of gravity.

For the completeness of the equations system (1)-(5) is used the following equality

$$
P_{k}\left(S_{w}\right)=P_{0}-P_{w}, S_{w}+S_{0}=1 \text {, }
$$

where $P_{k}$ is the capillary pressure.

For the single-valued definition of the pressure fields and saturation of the phases from the equations system (1) - (6) are complemented by initial and marginal conditions, which describes the initial state of the media and interrelation of the media with the environment. Marginal conditions are set regarding pressure or liquid discharge to wells and in the external border of the media. Usually, wells are modeled by using point sources (sewage), which are defined by the Dirac function, except for single-well system in the cylindrical coordinates [3]. It is important to note that, given approach resulted from the smallness of the well radius in comparison with the measures of the oil field. However, for the marginal conditions in the wells, it is essential to note the main circumstance. Traditionally, in the ground from where water - the displaced phase flows to the field (displacement wells, feed contour, gallery) either charge of the displaced phase or pressure is considered to be given. However, conditions given in exploitation wells generally are not satisfied. The working mode of exploitation wells, i.e., their flow and downhole pressure are set in dependency from the conditions of the system "media - well", also in the result of interrelation of wells. By this reason, it is practically impossible to describe conditions in exploitation wells precisely. In this regard, for practice in the development of oil fields modeling of the processes for the displacement of oil from the media by water on the base of information only from injection wells is very important. 


\section{Statement of the problem and solution method}

Let's assume that, is investigated non-deformable oil-producing media with the constant power $\mathrm{H}$, which horizontally located in a rectangular field $\Omega=\left\{0 \leq x \leq l_{x}, \quad 0 \leq y \leq l_{y}\right\}$. Roof and sole, and the lateral external border of the media is considered to be impenetrable for liquids. In the media, there are $\angle$ injected and as many exploitation wells. In the time moment $t=0$ through injected wells, water is pumped into the media under pressure, which exceeds the pressure of the media. It is assumed that during the displacement process in media is generated insulated double-phased flow of incompressible and mutually immiscible liquids - oil and water, subject to generalized law of Darcy. Because sizes of the wells are much less size of media, it is possible to neglect sizes of the wells by representing them as point flows (sources) with the capacities equal to the charges of real wells. By representing wells as point flows (sources) which are described by Dirac function, the mathematical model of the double-phased process in media excluding gravitational forces can be provided as

$$
\begin{gathered}
\phi \frac{\partial S_{w}}{\partial t}=\frac{\partial}{\partial x}\left(\frac{k k_{w}\left(S_{w}\right)}{\mu_{w}} \frac{\partial P_{w}}{\partial x}\right)+\frac{\partial}{\partial y}\left(\frac{k k_{w}\left(S_{w}\right)}{\mu_{w}} \frac{\partial P_{w}}{\partial y}\right)+\sum_{v=1}^{L} \frac{q_{w, v}(t)}{H} \delta\left(x-x_{v}^{w}, y-y_{v}^{w}\right), \\
\phi \frac{\partial S_{o}}{\partial t}=\frac{\partial}{\partial x}\left(\frac{k k_{o}\left(S_{w}\right)}{\mu_{w}} \frac{\partial P_{o}}{\partial x}\right)+\frac{\partial}{\partial y}\left(\frac{k k_{w}\left(S_{w}\right)}{\mu_{w}} \frac{\partial P_{o}}{\partial y}\right)-\sum_{v=1}^{L} \frac{q_{o, v}(t)}{H} \delta\left(x-x_{v}^{o}, y-y_{v}^{o}\right),
\end{gathered}
$$

where $\delta(\cdot)$-is Dirac function, $q_{o, v}(t),\left(x_{v}^{o}, y_{v}^{o}\right), v=\overline{1, L}$-are flows and coordinates of exploitation wells, $q_{w, v}(t),\left(x_{v}^{w}, y_{v}^{w}\right), \quad v=1, L$ - are flows and coordinates of injected wells.

By neglecting capillary pressure, i.e., assuming $P_{0}=P_{w}=P$ and denoting $S=S_{w}$, $\lambda(S)=k k_{\mathrm{w}}(S) / \mu_{\mathrm{w}}+k k_{o}(S) / \mu_{o}, \lambda_{\mathrm{w}}(S)=k k_{\mathrm{w}}(S) / \mu_{\mathrm{w}}$, the least system can be presented by the following type

$$
\begin{gathered}
\frac{\partial}{\partial x}\left(\lambda(S) \frac{\partial P}{\partial x}\right)+\frac{\partial}{\partial y}\left(\lambda(S) \frac{\partial P}{\partial y}\right)=\sum_{v=1}^{K} \frac{q_{o, v}(t)}{H} \delta\left(x-x_{v}^{o}, y-y_{v}^{o}\right)-\sum_{v=1}^{L} \frac{q_{w, v}(t)}{H} \delta\left(x-x_{v}^{w}, y-y_{v}^{w}\right), \\
\phi \frac{\partial S}{\partial t}=\frac{\partial}{\partial x}\left(\lambda_{w}(S) \frac{\partial P}{\partial x}\right)+\frac{\partial}{\partial y}\left(\lambda_{w}(S) \frac{\partial P}{\partial y}\right)+\sum_{v=1}^{L} \frac{q_{w, v}(t)}{H} \delta\left(x-x_{v}^{w}, \quad y-y_{v}^{w}\right), \\
(x, y) \subset \Omega, \quad 0<t \leq T .
\end{gathered}
$$

Let's assume that, in the initial time moment $t=0$ distribution of the pressure, and distribution of water saturation in the media are presented, i.e., for the system (7), (8) are defined following initial conditions

$$
\begin{gathered}
P(x, y, 0)=\varphi(x, y), \\
S(x, y, 0)=\psi(x, y) .
\end{gathered}
$$

Since the external border of the media is considered impenetrable, for the system (7), (8) will be defined following marginal conditions in external media:

$$
\frac{\partial P(0, y)}{\partial x}=\frac{\partial P\left(l_{x}, y\right)}{\partial x}=0, \frac{\partial P(x, 0)}{\partial y}=\frac{\partial P\left(x, l_{y}\right)}{\partial y}=0 .
$$

Flows of all injected wells $q_{w, v}(t) \quad v=\overline{1, L}$ are also considered to be given. However, related to the impossibility of regularization of oil collection in exploitation wells according to pre-determined program, their flows $q_{o, v}(t) v=\overline{1, L}$, are considered to be 
unknown and should be defined with the functions $S(x, y, t)$ and $P(x, y, t)$. It is clear that for the correct problem statement, it is necessary to set additional conditions. Let's assume that, as well as the flows of the wells at the same moment, is given change rule of downhole pressure over time in all injected wells. Then as additional conditions, we will have

$$
P\left(x_{v}^{w}, y_{v}^{w}, t\right)=\theta_{v}(t), v=\overline{1, L} .
$$

By this way, the problem is concluded as a definition of the functions $P(x, y, t)$ $S(x, y, t)$, and $q_{o, v}(t), v=\overline{1, L}$ which satisfies equations system (7), (8) and conditions (9)-(12). The objective relates to the class of inverse problems, which are related to the reconstruction of the right parts of the differential equations with the partial derivatives [4 - 5]. Statement issues and numerical methods for the solution of inverse problems for the reconstruction of the right parts of differential equations with the partial derivatives are investigated in [4-10].

For the solution of the problem (7)-(12) firstly let's include continuous difference scheme in the interval $[0 \leq t \leq T]$ for the variable $t$

$$
\bar{\omega}_{t}=\left\{t_{j}=j \Delta t, j=\overline{0, m}\right\}
$$

with the step $\Delta t=T / m$. The derivative $\frac{\partial S(x, y, t)}{\partial t}$ in the equation (8) we can discretize with the difference "back" when $t_{j}, j=\overline{1, m}$

$$
\left.\frac{\partial S(x, y, t)}{\partial t}\right|_{t=t_{j}} \approx \frac{S\left(x, y, t_{j}\right)-S\left(x, y, t_{j-1}\right)}{\Delta t}
$$

also, obtained differential difference task for each fixed value $j, j=1,2, \ldots, m$ fragmenting it off into two consequentially solvable subproblems, we can write as

$$
\begin{gathered}
\frac{\partial}{\partial x}\left(\lambda\left(S^{j-1}\right) \frac{\partial P^{j}(x, y)}{\partial x}\right)+\frac{\partial}{\partial y}\left(\lambda\left(S^{j-1}\right) \frac{\partial P^{j}(x, y)}{\partial y}\right)= \\
=\sum_{v=1}^{L} \frac{q_{o, v}^{j}}{H} \delta\left(x-x_{v}^{o}, y-y_{v}^{o}\right)-\sum_{v=1}^{L} \frac{q_{w, v}^{j}}{H} \delta\left(x-x_{v}^{w}, y-y_{v}^{w}\right) \\
(x, y) \subset \Omega, \\
\frac{\partial P^{j}(0, y)}{\partial x}=\frac{\partial P^{j}\left(l_{x}, y\right)}{\partial x}=0, \frac{\partial P^{j}(x, 0)}{\partial y}=\frac{\partial P^{j}\left(x, l_{y}\right)}{\partial y}=0, \\
P^{j}\left(x_{v}^{w}, y_{v}^{w}\right)=\theta_{v}^{j}, v=\overline{1, K} . \\
+\frac{\partial}{\partial y}\left(\lambda_{w}\left(S^{j-1}\right) \frac{\partial P^{j}(x, y)}{\partial y}\right)+\sum_{v=1}^{L} \frac{q_{w, v}^{j}}{H} \delta\left(x-x_{v}^{w}, y-y_{v}^{w}\right) \\
(x, y) \subset \Omega, \\
j=1,2, \ldots, m, \\
P^{0}(x, y)=\varphi(x, y), \\
S^{0}(x, y)=\psi(x, y),
\end{gathered}
$$


$S^{j}(x, y) \approx S\left(x, y, t_{i}\right), P^{j}(x, y) \approx P\left(x, y, t_{i}\right) q_{w v}^{j}=q_{w v}\left(t_{i}\right), q_{o . v}^{j} \approx q_{o . v}\left(t_{i}\right), \theta_{v}^{j}=\theta_{v}\left(t_{i}\right)$.

The first subproblem (13) - (15) is an inverse problem for the definition of the function $P^{j}(x, y)$ and variables $q_{o, v}^{j}, \quad v=\overline{1, L}$. Nevertheless, the function $S^{j-1}(x, y)$ is considered to be known. The second subproblem is the solution of the equation (16) regarding the unknown function $S^{j}(x, y)$.

Now let's assume that the solution of the subproblem (13)-(15) for each fixed value $j, j=1,2, \ldots, m$ can be represented as [7-11]:

$$
P^{j}(x, y)=\sum_{v=1}^{L} p_{v}^{j}(x, y) q_{o, v}^{j}+p_{0}^{j}(x, y)
$$

where $p_{0}^{j}(x, y) p_{v}^{j}(x, y), v=\overline{1, L}$ - are unknown functions. By setting equality (19) in (13), (14) we will have

$$
\begin{gathered}
\sum_{v=1}^{K} q_{o, v}^{j}\left[\frac{\partial}{\partial x}\left(\lambda\left(S^{j-1}\right) \frac{\partial p_{v}^{j}}{\partial x}\right)+\frac{\partial}{\partial y}\left(\lambda\left(S^{j-1}\right) \frac{\partial p_{v}^{j}}{\partial y}\right)-\frac{1}{H} \delta\left(x-x_{v}^{o}, y-y_{v}^{o}\right)\right]+ \\
+\left[\frac{\partial}{\partial x}\left(\lambda\left(S^{j-1}\right) \frac{\partial p_{0}^{j}}{\partial x}\right)+\frac{\partial}{\partial y}\left(\lambda\left(S^{j-1}\right) \frac{\partial p_{0}^{j}}{\partial y}\right)+\sum_{v=1}^{L} \frac{q_{w, v}^{j}}{H} \delta\left(x-x_{v}^{w}, y-y_{v}^{w}\right)\right]=0 \\
\sum_{v=1}^{K} q_{o, v}^{j} \frac{\partial p_{v}^{j}(0, y)}{\partial x}+\frac{\partial p_{0}^{j}(0, y)}{\partial x}=\sum_{v=1}^{K} q_{o, v}^{j} \frac{\partial p_{v}^{j}\left(l_{x}, y\right)}{\partial x}+\frac{\partial p_{0}^{j}\left(l_{x}, y\right)}{\partial x}=0 \\
\sum_{v=1}^{K} q_{o, v}^{j} \frac{\partial p_{v}^{j}(x, 0)}{\partial y}+\frac{\partial p_{0}^{j}(x, 0)}{\partial y}=\sum_{v=1}^{K} q_{o, v}^{j} \frac{\partial p_{v}^{j}\left(x, l_{y}\right)}{\partial y}+\frac{\partial p_{0}^{j}\left(x, l_{y}\right)}{\partial y}=0
\end{gathered}
$$

From the last expressions, it is possible to get boundary problems regarding $p_{0}^{j}(x, y), p_{v}^{j}(x, y), v=\overline{1, L}$

$$
\begin{gathered}
\frac{\partial}{\partial x}\left(\lambda\left(S^{j-1}\right) \frac{\partial p_{0}^{j}}{\partial x}\right)+\frac{\partial}{\partial y}\left(\lambda\left(S^{j-1}\right) \frac{\partial p_{0}^{j}}{\partial y}\right)=-\sum_{v=1}^{L} \frac{q_{w, v}^{j}}{H} \delta\left(x-x_{v}^{w}, y-y_{v}^{w}\right),(x, y) \subset \Omega, \\
\frac{\partial p_{0}^{j}(0, y)}{\partial x}=\frac{\partial p_{0}^{j}\left(l_{x}, y\right)}{\partial x}=0, \frac{\partial p_{0}^{j}(x, 0)}{\partial y}=\frac{\partial p_{0}^{j}\left(x, l_{y}\right)}{\partial y}=0 . \\
\frac{\partial}{\partial x}\left(\lambda\left(S^{j-1}\right) \frac{\partial p_{v}^{j}}{\partial x}\right)+\frac{\partial}{\partial y}\left(\lambda\left(S^{j-1}\right) \frac{\partial p_{v}^{j}}{\partial y}\right)=\frac{1}{H} \delta\left(x-x_{v}^{o}, y-y_{v}^{o}\right),(x, y) \subset \Omega, \\
\frac{\partial p_{v}^{j}(0, y)}{\partial x}=\frac{\partial p_{v}^{j}\left(l_{x}, y\right)}{\partial x}=0, \frac{\partial p_{v}^{j}(x, 0)}{\partial y}=\frac{\partial p_{v}^{j}\left(x, l_{y}\right)}{\partial y}=0 .
\end{gathered}
$$

As we can see problems for definition of the functions $p_{0}^{j}(x, y), p_{v}^{j}(x, y), v=\overline{1, L}$ (20), (21) and (22), (23) are independent from the variables $q_{o, v}^{j}, v=1, L$ and can be parallelized. Let's assume that, problem (20), (21) and problem (22), (23) are solved for each value $v=\overline{1, L}$, i.e. functions $p_{0}^{j}(x, y) \backsim p_{v}^{j}(x, y), v=\overline{1, L}$ are defined. Then setting expression (19) in the additional conditions (15) we obtain following linear algebraic system of equations regarding to unknown variables $q_{o . v}^{j}, v=\overline{1, L}$ :

$$
\begin{aligned}
& \sum_{v=1}^{L} p_{v}^{j}\left(x_{1}^{w}, y_{1}^{w}\right) q_{o, v}^{j}=\theta_{1}^{j}-p_{0}^{j}\left(x_{1}^{w}, y_{1}^{w}\right) \\
& \sum_{v=1}^{L} p_{v}^{j}\left(x_{2}^{w}, y_{2}^{w}\right) q_{o, v}^{j}=\theta_{2}^{j}-p_{0}^{j}\left(x_{2}^{w}, y_{2}^{w}\right)
\end{aligned}
$$




$$
\sum_{v=1}^{L} p_{v}^{j}\left(x_{K}^{w}, y_{K}^{w}\right) q_{o, v}^{j}=\theta_{K}^{j}-p_{0}^{j}\left(x_{K}^{w}, y_{K}^{w}\right)
$$

It is clear that, by the solution of the equations system (24) and definition of $q_{o, v}^{\prime}$ $v=\overline{1, L}$, it is possible to obtain the function $P^{j}(x, y)$ with the formula (19). Then by setting $P^{j}(x, y)$ in the equation (16), it is possible to find $S^{j}(x, y)$.

In this way computing algorithm for solution of difference-differential problem (7)(12) for definition of $P^{j}(x, y), S^{j}(x, y) \quad$ и $q_{o, v}^{\prime}, \quad v=\overline{1, L}$ for each fixed value $j=1,2, \ldots, m$ established by parallel solution of forward difference-differential problems (14), (15) and (16), (17) regarding to the functions $p_{0}^{j}(x, y), p_{v}^{j}(x, y)$, $v=\overline{1, L}$, definition of $q_{o, v}^{\prime}, v=\overline{1, L}$ from the algebraic linear equations system (18), using expression (13) for $P^{j}(x, y)$ and solution of the equation (10) for the function $S^{j}(x, y)$.

By this way, the proposed parallel numerical method enables to define sequentially flows of exploitative wells, distribution of the pressure in the media, and distribution of the saturation of displaced phase.

\section{Experimental results}

For clarifying effectivity of the application of the proposed computational algorithm were provided numerical experiments for model problems. The calculation was carried out in space-time difference network with the steps $h=0.1, \tau=0.01$. For parallelization of calculation, processes was applied OpenMP parallel programming technology. Results of the numerical experiments providing for the case, when $\mu_{w}=1 \cdot 10^{-3}$ Pa.s, $\mu_{o}=1 \cdot 10^{-3}$ Pa.s, $k=0.5 \cdot 10^{-12} \mathrm{~m}^{2}$,

$$
k_{w}(S)=\left(\frac{S-0.2}{0.8}\right)^{3}, \quad k_{o}(S)=\left(\frac{0.8-S}{0.6}\right)^{3}, \phi=0.3,
$$

$l_{x}=2000 \mathrm{~m}, \quad l_{y}=2000 \mathrm{~m}, \quad \varphi(x, y)=100 \cdot 10^{5} \mathrm{~Pa}, \quad \psi(x, y)=0, \quad L=4, q_{w, 1}(t)=24 \mathrm{~m}^{3} /$ day., $q_{w .2}(t)=30 \mathrm{~m}^{3} /$ day., $\theta_{1}(t)=120 \cdot 10^{5} \mathrm{~Pa}, \theta_{2}(t)=122 \cdot 10^{5} \mathrm{~Pa}$ are described in table 1 .

Numerical results of the calculations show that volume of the saturated phase on given time is equal to the produced oil in the exploitation wells on this time.

Results analysis of the numerical experiments justifies that, the proposed numerical method can be used for predicting the state of medium system exploited by the water flooding method.

\section{Table 1. Differences between running times of sequential and parallel algorithms}

\begin{tabular}{|c|c|c|c|}
\hline $\mathrm{N}$ & Length of the step, $\mathrm{h}$ & $\begin{array}{c}\text { Running time for } \\
\text { sequential algorithm } \\
\text { (seconds) }\end{array}$ & $\begin{array}{c}\text { Running time for paral- } \\
\text { lel algorithm (seconds) }\end{array}$ \\
\hline 1 & 0.2 & 232.51 & 98.37 \\
\hline 2 & 0.1 & 557.37 & 236.47 \\
\hline 3 & 0.05 & 1980.370 & 805.81 \\
\hline
\end{tabular}




\section{References}

[1] Masket, M. (2004). Techenie odnorodnyh zhidkostej v poristoj srede. MoskvaIzhevsk: Institut komp'juternyh issledovanij, 628.

[2] Konovalov, A. N. (1988). Zadachi fil'tracii mnogofaznoj neszhimaemoj zhidkosti. Nauka. Sib. otd-nie.

[3] Aziz, H., Settari, Je., Korolev, A. V., Kestner, V. P., \& Maksimov, M. M. (2004). Matematicheskoe modelirovanie plastovyh sistem. Reguljarnaja i haoticheskaja dinamika| Izhevskij institut komp'juternyh issledovanij.

[4] Samarskij, A. A., \& Vabishchevich, P. N. (2004). Chislennye metody reshenija obratnyh zadach matematicheskoj fiziki. URSS.

[5] Borukhov, V. T., \& Vabishchevich, P. N. (2000). Numerical solution of the inverse problem of reconstructing a distributed right-hand side of a parabolic equation. Computer physics communications, 126(1-2), 32-36.

[6] Vabishchevich, P. N., Vasil'ev, V. I., \& Vasil'eva, M. V. (2015). Vychislitel'naja identifikacija pravoj chasti parabolicheskogo uravnenija. Zhurnal vychislitel'noj matematiki i matematicheskoj fiziki, 55(6), 1020-1027.

[7] Vabishchevich, P. N., Vasil'ev, V. I., Vasil'eva, M. V., \& Nikiforov, D. Ja. (2015). Chislennoe reshenie odnoj obratnoj zadachi fil'tracii. Uchenye zapiski Kazanskogo universiteta. Serija Fiziko-matematicheskie nauki, 157(4).

[8] Gamzaev, K. M. (2015). Modeling nonstationary nonlinear-viscous liquid flows through a pipeline. Journal of Engineering Physics and Thermophysics, 88(2), 480485.

[9] Gamzaev, K. M. (2017). Numerical solution of combined inverse problem for generalized Burgers equation. Journal of Mathematical Sciences 221 (6), 833-839.

[10] Deng, Z. C., Qian, K., Rao, X. B., Yang, L., \& Luo, G. W. (2015). An inverse problem of identifying the source coefficient in a degenerate heat equation. Inverse Problems in Science and Engineering, 23(3), 498-517.

[11] Marchuk, G. I. (1982). Matematicheskoe modelirovanie $\vee$ probleme okruzhajushhej sredy. " Nauka," Glav. red. fiziko-matematicheskoj lit-ry. 